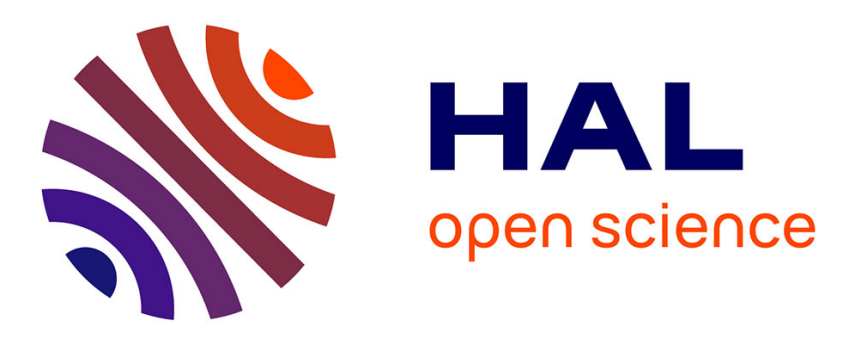

\title{
Proposition d'un outil de repérage de facteurs de vulnérabilité des salariés par l'infirmier en Santé au travail
}

S. Nahum-Henry, A. Descatha

\section{- To cite this version:}

S. Nahum-Henry, A. Descatha. Proposition d'un outil de repérage de facteurs de vulnérabilité des salariés par l'infirmier en Santé au travail. Archives des Maladies Professionnelles et de L'Environnement, 2021, 82 (2), pp.216-229. 10.1016/j.admp.2020.08.004 . hal-03122719

\section{HAL Id: hal-03122719 \\ https://hal.science/hal-03122719}

Submitted on 5 Feb 2021

HAL is a multi-disciplinary open access archive for the deposit and dissemination of scientific research documents, whether they are published or not. The documents may come from teaching and research institutions in France or abroad, or from public or private research centers.
L'archive ouverte pluridisciplinaire HAL, est destinée au dépôt et à la diffusion de documents scientifiques de niveau recherche, publiés ou non, émanant des établissements d'enseignement et de recherche français ou étrangers, des laboratoires publics ou privés. 


\section{PAGE DE TITRE}

Proposition d'un outil de repérage de facteurs de vulnérabilité des salariés par l'infirmier en Santé au travail

Proposal for a tool for identifying vulnerability factors by the occupational health nurse

$\underline{\text { RUBRIQUE }}$ Mise au point

Auteur de correspondance: S Nahum-Henry, ACMS 55 rue Rouget de Lisle 92150 Suresnes

Sidonie Nahum-Henry 130 avenue du général de Gaulle 92130 Issy les Moulineaux

nahumsido@gmail.com

A Descatha, Université d'Angers, CHU Angers, Univ Rennes, Inserm, EHESP, Irset, UMR_S1085, Angers, France 


\section{POINTS ESSENTIELS}

- La fragilité représente un réel enjeu de Santé publique.

- La communauté médicale gériatrique s'intéresse au repérage précoce de la fragilité chez les personnes âgées afin d'envisager des actions de prévention avant le stade irréversible de la dépendance.

- La Société Française de Gériatrie et de Gérontologie (SFGG) a adopté en 2011 la définition suivante de la fragilité :

«La fragilité est un syndrome clinique. Il reflète une diminution des capacités physiologiques de réserve qui altère les mécanismes d'adaptation au stress. Son expression clinique est modulée par les comorbidités et des facteurs psychologiques, sociaux économiques et comportementaux. Le syndrome de fragilité est un marqueur de risque de mortalité et d'événements péjoratifs, notamment d'incapacités, de chutes, d'hospitalisation et d'entrée en institution. L'âge est un déterminant majeur de fragilité mais n'explique pas à lui seul ce syndrome. La prise en charge des déterminants de la fragilité peut réduire ou retarder ses conséquences. Ainsi, la fragilité s'inscrirait dans un processus potentiellement réversible » (Rolland, 2011).

- Le modèle de phénotype de fragilité de Linda Fried est validé et détermine cinq critères (perte de poids involontaire, sensation de fatigue voire d'épuisement, diminution de la force de préhension, ralentissement de la marche, activité physique réduite) permettant un classement en trois états facilement repérables (pas de critère/ robuste, 1 à 2 critères/ préfragile, 3 critères ou plus/ fragile).

- En médecine du travail, la transposition de ce concept de fragilité permettrait le repérage des salariés vulnérables par l'infirmier en santé au travail lors de la visite d'information et de prévention et une orientation pertinente vers le médecin du travail afin de mettre en place un plan d'action, de sensibilisation et de prévention. 


\section{Summary}

Introduction: The purpose of this study is to reflect on the interest of transposing the concept of frailty developed in Geriatrics into Occupational Medicine to allow identification of "at risk" employees during the visit made by the occupational health nurses.

Method: Starting from the definition of the concept of frailty in Geriatrics and two researches on frailty of 50 years and over, the SHARE survey and the HEAF study, we applied the criteria identified on three clinical situations encountered in an occupational health service.

Results: We observe that the identification of frailty and the implementation of corrective actions allow an improvement in the quality of life and felt comfort and a prevention of occupational exclusion.

Discussion: The concept of frailty therefore appears to be transposable to the younger subject and could in particular help to sensitize certain healthcare physicians to a global care and certain public health decision-makers to the importance of the preventive visit for 45-50 years old. Basically, this concept is already applied in Occupational Medicine, which is concerned not only with biomedical determinants but also with environmental ones. Following the detection of vulnerability factors, the occupational physician can intervene on the professional environment to adapt work to humans.

Conclusion: Ultimately, the development of a simple tracking tool could be made available to occupational health nurses to allow suitable and relevant referral to the occupational physician. 


\section{$\underline{\text { Résumé }}$}

Introduction : L'objet de ce travail est de réfléchir à l'intérêt d'une transposition en Médecine du Travail du concept de fragilité développé en Gériatrie pour permettre un repérage des salariés à risque lors de la visite réalisée par l'infirmier(e) en santé au travail.

Méthode : Partant de la définition du concept de fragilité en gériatrie et de deux travaux sur la fragilité des 50 ans et plus, l'enquête SHARE et l'étude HEAF, nous avons appliqué les critères dégagés à trois situations cliniques rencontrées dans un service de santé au travail.

Résultats: Nous observons que le repérage de la fragilité et la mise en place d'actions correctrices permettent une amélioration de la qualité de vie et du confort ressenti et une prévention de la désinsertion professionnelle.

Discussion: Le concept de fragilité parait donc transposable chez le sujet plus jeune et pourrait notamment contribuer à sensibiliser certains médecins de soins à une prise en charge globale et certains décideurs en Santé Publique à l'importance de la visite préventive des 45 50 ans. Au fond, ce concept est déjà appliqué en Médecine du Travail celle-ci s’intéressant non seulement aux déterminants biomédicaux mais aussi aux déterminants environnementaux. Suite à la détection de facteurs de vulnérabilité, le médecin du travail peut intervenir sur l'environnement professionnel pour adapter le travail à l'homme.

Conclusion : A terme, le développement d'un outil simple de repérage pourrait être mis à disposition des infirmier(e)s en santé au travail pour permettre une orientation adaptée et pertinente vers le médecin du travail.

Mots clés : Fragilité; vulnérabilité; Outil de repérage; Prévention; Santé au travail

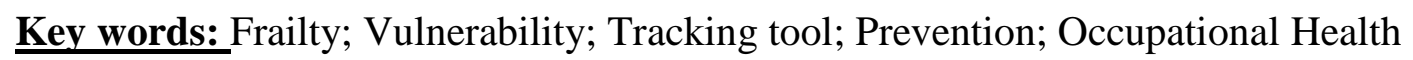




\section{CORPS DE TEXTE}

\section{INTRODUCTION}

Depuis une vingtaine d'années, la communauté médicale gériatrique s'intéresse au repérage des personnes âgées fragiles, à la recherche des causes de fragilité et des actions à mettre en place, afin de prévenir la dépendance et de discuter les propositions de thérapeutiques adaptées au contexte clinique. Entre le vieillissement normal et pathologique, les anglosaxons décrivent un vieillissement intermédiaire qu'ils nomment « frailty » ou fragilité. Sa définition la plus simple correspond à «l'impossibilité de répondre de façon adaptée à un stress qu'il soit médical, psychologique ou social».

Dans le cadre de la modernisation de la médecine du travail, le décret d'application $\mathrm{n}^{\circ} 2016$ 1908 publié au JO du 27 décembre 2016, précise de nouvelles modalités de suivi individuel de l'état de santé du travailleur. En pratique, la visite d'information et de prévention peut être réalisée dans le cadre du suivi individuel simple par un(e) infirmier(e) en santé au travail, et un des enjeux de cette visite est de définir et repérer les situations à risque et de réadresser le salarié vers le médecin du travail de façon pertinente.

L'objet de cet article est de réfléchir à l'intérêt d'une transposition en Médecine du_Travail du concept de fragilité développé en Gériatrie pour faciliter le repérage des salariés à risque lors de la visite réalisée par l'infirmier(e) en santé au travail.

\section{PREAMBULE : DEFINITION DU CONCEPT DE FRAGILITE EN GERIATRIE}

Selon Linda P. Fried, « la fragilité est une vulnérabilité liée à l'avancée en âge, due à une altération des réserves homéostatiques de l'organisme qui devient incapable de surmonter un quelconque stress.» [1] Malgré la proposition d'innombrables définitions et de plus de 70 échelles pour évaluer l'état de fragilité, les 5 critères de L. Fried restent les plus utilisés : 
- La perte de poids involontaire,

- La sensation de fatigue voire d'épuisement,

- La diminution de la force de préhension,

- Le ralentissement de la marche,

- L'activité physique réduite.

Le phénotype de fragilité se définit par 3 des critères susmentionnés ou plus, alors qu'un état de pré-fragilité correspond à 2 de ces critères. [3] [figure 1]

Le concept de fragilité peut se concevoir comme un stade intermédiaire entre le vieillissement habituel ou usuel (usual aging), avec des atteintes considérées comme physiologiques, liées à l'âge, de certaines fonctions, mais sans pathologie bien définie et le vieillissement pathologique avec pathologies sévères évolutives ou compliquées et/ou handicaps (pathological aging). Ce terme suggère un état d'instabilité avec risque de perte fonctionnelle ou de majoration de la perte fonctionnelle existante. L'incapacité (disability) du sujet n'est pas installée, mais peut survenir à l'occasion d'une pathologie aiguë ou d'événements extérieurs, même minimes. [1] [4] [figure 2]

\section{OBJECTIF}

Dans le cadre de la modernisation de la Médecine du_Travail, l'infirmier(e) en santé au travail peut réaliser les visites d'information et de prévention initiales et périodiques dans le respect du protocole de délégation - en pratique quotidienne actuelle, la question de la réorientation pertinente du salarié vers le médecin du travail est centrale, ce d'autant plus que, devant la pénurie chronique de médecins du travail et les demandes qui leur sont faites, la planification des visites réalisées par ceux-ci nécessite le meilleur aiguillage possible. 
Etant donné l'augmentation prévisible des années de vie professionnelle, on peut supposer que le processus de vieillissement va être influencé par des facteurs liés au travail, qui pourraient déclencher ou accélérer l'entrée en fragilité des salariés au fur et à mesure que leurs âges augmentent.

Nous nous sommes donc demandés si une transposition en Médecine-du Travail du concept de fragilité développé en Gériatrie ne pourrait pas être une aide au repérage des salariés à risque lors de la visite réalisée par l'infirmier(e) en santé au travail et ainsi permettre une réorientation adaptée.

De plus, le syndrome de fragilité possédant à la fois un caractère dynamique et évolutif, et étant un marqueur de risque d'événements péjoratifs, sa détection ne pourrait-elle pas être un axe de prévention à exploiter en Médecine du_Travail?

\section{METHODE}

Partant de la définition du concept de fragilité en gériatrie présenté en préambule, nous nous sommes intéressés aux deux principaux travaux réalisés sur la fragilité des 50 ans et plus, l'enquête SHARE et l'étude HEAF, et avons tenté d'identifier les corrélations possibles avec la fragilité au travail.

Nous nous sommes appuyés notamment sur les critères dégagés dans ces études pour les appliquer à trois situations cliniques rencontrées au sein d'une collectivité d'un service interentreprises de santé au travail de la région parisienne afin de repérer la fragilité du salarié lors de la visite d'information et de prévention réalisée par l'infirmier(e) en santé au travail pour permettre l'orientation vers le médecin du travail puis apprécier son évolution en fonction d'actions correctives mises en place. 


\section{Principaux travaux sur la fragilité chez les $\mathbf{5 0}$ ans et plus}

\subsection{L'enquête européenne SHARE (Survey on Health, Ageing and Retirement in Europe)}

\subsubsection{Présentation}

C'est une enquête longitudinale, pluridisciplinaire et internationale concernant plus de 80000 européens âgés de 50 ans et plus. L'enquête est réalisée tous les deux ans depuis 2004 et se déroule dans vingt-sept pays européens. Les données recueillies portent sur l'état de santé des répondants et de leurs proches ainsi que sur leur situation sociale (famille, entraide, réseaux sociaux) et économique (emploi, retraite, patrimoine).

L'objectif principal est d'obtenir des données sur l'état de santé, le vieillissement et la retraite auprès d'un échantillon représentatif de la population des plus de 50 ans.

\subsubsection{Méthode}

L'enquête a pour objectif d'analyser les problèmes économiques et sociaux liés au vieillissement de la population en Europe. La cohorte s'articule autour de trois grands thèmes : santé, économie et sociologie.

C'est une enquête longitudinale qui a pour objectif d'analyser des données individuelles sur la durée et pour cela elle réinterroge tous les deux ans les personnes déjà interrogées lors des vagues précédentes, en plus d'éventuels rafraichissements de l'échantillon initial, avec application de la définition de la fragilité.

Son caractère pluridisciplinaire permet d'analyser des questions importantes liées au vieillissement et, en particulier : 
1) la santé au travail des seniors (50-64 ans), les causes et les conséquences du passage à la retraite ;

2) Les relations intergénérationnelles, les solidarités familiales, les activités sociales (6580 ans) ;

3) Les modalités de prise en charge de la dépendance, la santé mentale (80 ans et plus).

La première vague a été lancée en 2004 et l'enquête devrait se poursuivre jusqu'en 2024. La construction d'un panel longitudinal permet de disposer d'une image complète du processus de vieillissement et des interactions complexes entre facteurs sociaux, économiques et de santé dans une perspective dynamique.

\subsubsection{Résultats}

L'application de la définition de la fragilité à l'échelle européenne à 18.227 participants de plus de 50 ans, vivant à domicile, a permis d'estimer qu'en France la prévalence de la fragilité était de $15 \%$ et celle de la pré-fragilité de 43,6\%. [5] [6]

Le suivi à 2 ans (2004-2006) de 11.015 personnes âgées de plus de 60 ans, vivant dans la communauté et classifiées comme fragiles ou pré-fragiles dans l'étude SHARE atteste de plusieurs faits :

- Une altération de la mobilité - difficultés auto-rapportées pour rester assis 2 heures, se mettre debout d'une chaise sans l'aide des mains, monter un étage sans s'arrêter pour se reposer, élever les membres au-dessus des épaules, soulever un poids de $5 \mathrm{~kg}$.

- Une dégradation des activités de base de la vie quotidienne (hygiène corporelle, habillage, aller aux toilettes, locomotion, continence, repas): évaluée avec l'échelle ADL (annexe 1)

- Une altération des activités instrumentales de la vie quotidienne (aptitude à utiliser le téléphone, courses, préparation des aliments, entretien ménager, blanchisserie, moyens de 
transport, responsabilité à l'égard de son traitement, aptitude à manipuler l'argent) : évaluée par l'échelle IADL (annexe 2)

- Une augmentation des comorbidités. [7]

Les aggravations observées sont significatives tant chez les personnes fragiles que chez les pré-fragiles, même si l'amplitude de l'aggravation est moindre pour ces derniers.

Le suivi entre 2006 et 2011 de 15.566 participants démontre que les états de pré-fragilité et de fragilité sont réversibles. Ainsi, entre 2006 et 2011, 51,4\% des pré-fragiles sont restés stables, $32,4 \%$ sont redevenus robustes alors que $11,6 \%$ étaient devenus fragiles/dépendants et 4,6\% étaient morts. La même observation est faite pour les fragiles qui retournent à l'état de préfragiles dans $31,9 \%$ des cas et à celui de robuste dans $7 \%$ des cas. [8]

A noter qu'aucune intervention n'était prévue dans cette étude, mais il est fort possible que certains individus se soient spontanément occupés de leur santé en améliorant leurs apports nutritionnels et leur activité physique, mais cette étude européenne ne cible pas cet aspect.

\subsection{L'etude HEAF (Health and Employment After Fifty)}

\subsubsection{Présentation}

C'est une étude de cohorte sur l'impact potentiel du phénotype fragilité sur le travail après 50 ans dans laquelle les critères de «Fried and al » sont utilisés. Cela intègre la perte de poids involontaire, l'épuisement auto-déclaré, la marche ralentie, un niveau d'activité physique faible et la faiblesse de la préhension.

Il a été recherché :

1) si la fragilité, définie par ces critères, est associée à des effets négatifs sur l'emploi : perte d'emploi, perte de travail liée à la santé, arrêt maladie de longue durée, réduction importante d'activité et difficulté à assurer le travail ; 
2) quelle est la contribution de la fragilité à ces effets négatifs ;

3) si les résultats sont affectés par l'activité des personnes

4) si certains critères sont corrélés de manière plus importante que d'autres avec les difficultés au travail.

\subsubsection{Méthode}

L'étude de cohorte HEAF est intégrée à la Clinical Practice Research Datalink (CPRD), une base de données Britannique sur les soins primaires.

En résumé, en 2013-2014, un échantillon aléatoire de patients nés entre 1948 et 1962 a été constitué (âge cible 50-64 ans) enregistrés auprès de 24 cabinets de médecine générale anglais pour constituer la base de données du CPRD. Un questionnaire a été envoyé à 39359 personnes $(97.5 \%$ de ceux qui ont été identifiés à l'exception des malades ou des récemment décédés ou de ceux qui ont été désinscrits entre le moment de la sélection et de l'envoi du questionnaire). En tout, 8.134 personnes ont renvoyé un questionnaire valide, ce qui a permis d'être pertinent pour l'analyse qui portait sur les fonctions physiques, la santé et les critères d'emploi.

Pour mettre en évidence la fragilité, les critères de Fried ont été utilisés et adaptés :

- Perte de poids : au cours des 12 derniers mois, perte de poids de plus de 4,5 kg non volontaire, i.e. sans régime et exercices (oui/non)

- Epuisement auto-déclaré : dans les 7 jours précédents, tout geste est effort ou ne pas être capable de se mobiliser (l'un des 2 critères ou les deux tout le temps ou presque)

- Marche ralentie : vitesse de marche habituelle décrite comme difficile ou très lente 
- Faiblesse dans la force de préhension : difficultés sévères ou modérées pour ouvrir un bocal n’ayant jamais été ouvert auparavant.

- Niveau d'activité faible: au cours d'une semaine habituelle, et en dehors du travail rémunéré, ne pas avoir fait d'activité physique suffisante pour entrainer une sudation.

Les questions relatives à l'emploi portaient sur :

- le statut professionnel actuel et dans le cas de l'absence d'emploi si le travail précédent avait été quitté pour raisons de santé:

- la profession ou dernière profession occupée (classement en fonction de la classification standard professionnelle 2010 -SOC 2010 -en manager supérieur, profession intermédiaire ou emploi routinier ou manuel);

- le nombre de jours d'absence au cours des 12 derniers mois ;

- la nécessité de réduire sa charge de travail pour raison de santé dans les 12 derniers mois ;

- l'adaptation actuelle aux exigences physiques ou psychologiques liées au travail ;

- la capacité physique et psychologique déclarée de faire le même type de travail dans les 2 ans ;

- la perception de la sécurité de l'emploi ;

- en cas d'insomnies si la cause en était une inquiétude ou une difficulté liée au travail.

Des informations ont également été collectées en combinant plusieurs associations, à savoir :

- faible bien-être, selon les 14 items de l'échelle du Warwick Edimburgh Mental Well Being (WEMWBS), annexe 3; 
- douleur persistante pendant un mois ou plus dans les 12 derniers mois affectant le dos ou le cou, les bras ou les épaules ou les jambes et qui rendent difficile voire impossible de se laver, de s'habiller ou de réaliser des travaux ménagers.

\subsubsection{Résultats}

Dans cette étude portant sur plus de 8.000 personnes âgées de 50 à 65 ans issues des cabinets anglais de médecine générale, les auteurs Palmer and al. [9] observent que près d'un tiers des personnes sont en situation de pré-fragilité et 3.9\% sont fragiles. La fragilité est fortement corrélée avec le fait d'être sans emploi, avec la perte de travail liée à la santé, les absences maladie prolongées, le fait d'avoir à réduire fortement son activité au travail, l'autodéclaration de ne pas assumer les exigences de son travail et la perception négative du travail incluant l'insécurité au travail et l'inquiétude liée au travail entraînant une perte de sommeil.

Ceux qui sont classés «fragiles » sont environ 5 fois plus nombreux à signaler un faible bienêtre et des douleurs persistantes, deux critères qui sont également des facteurs de risque de perte de travail liée à leur santé, d'absences pour maladie et de réduction des capacités de travail.

Les corrélations entre fragilité et effets négatifs du travail apparaissent comme étant associées principalement à une faible vitesse de marche, une faiblesse de la préhension et un épuisement suggérant que des fonctions motrices défaillantes peuvent être un important facteur sousjacent.

Les corrélations entre «être sans emploi », «prendre des congés maladie » et «mauvaise adaptation » sont plus fortes pour les professions routinières ou manuelles. Ce différentiel peut être dû à une mauvaise santé physique générale ou plus simplement à un plus grand décalage entre capacités physiques et exigences du travail - la capacité des travailleurs 
manuels à effectuer leur travail est plus facilement compromise par une détérioration de leurs capacités physiques.

Pour Palmer and al., les résultats ont besoin d'être corroborés par une étude longitudinale et les fonctions physiques et les mesures cliniques liées à de nombreux symptômes caractéristiques sont le possible prélude au développement d'instruments pour identifier la fragilité au travail.

Au final, ces deux études soulignent toute l'importance du repérage car c'est à ce stade que les interventions peuvent être les plus efficaces pour agir sur la pré-fragilité ou la fragilité qui sont des états instables mais partiellement ou totalement réversibles.

\section{Application des critères de fragilité dans trois situations cliniques rencontrées au sein d'une collectivité d'un service interentreprises de santé au travail de la région parisienne}

\subsection{Mme N}

Agée de 50 ans, elle occupe un poste d'agent des services hospitaliers dans une clinique de soins de suite et rééducation depuis mars 2014.

Elle a pour antécédent notable une drépanocytose homozygote $\mathrm{S} / \mathrm{S}$, responsable d'une anémie hémolytique chronique et de douleurs osseuses épisodiques dans le cadre de crises vasoocclusives, dont le traitement de fond est l'Hydroxycarbamide (Siklos ${ }^{\circledR}$ ) avec un suivi régulier par un hématologue hospitalier depuis 2009.

Les symptômes décrits lors de la visite périodique réalisée par l'infirmier en santé au travail, sont un épuisement physique, une marche ralentie, une absence d'activité physique, un blocage du pouce droit avec diminution de la force de préhension, des douleurs du poignet droit, des douleurs du genou droit, une anxiété liée à la crainte de la perte de son emploi. 
Ces symptômes ont motivé plusieurs arrêts maladie dans les derniers mois, dont une hospitalisation pour crise vaso-occlusive.

Elle remplit donc 4 critères selon la nomenclature de Fried, ce qui la classe comme « fragile ».

Dans ce contexte, elle est réadressée au médecin du travail afin d'évaluer la situation médicale et de mettre en place un plan d'action :

- lors du contact avec l'hématologue référent, celui-ci souligne que l'anémie étant responsable d'une fatigabilité à l'effort, il est important que des plages de repos soient respectées, qu'elle puisse s'hydrater régulièrement et qu'elle ne soit pas exposée au froid ou au chaud de façon trop prolongée pour la prévention des crises vasoocclusives.

- Un avis spécialisé auprès d'un rhumatologue est pris devant une suspicion de pouce à ressaut et une infiltration est réalisée, permettant d'améliorer la symptomatologie,

- La constitution d'un dossier auprès de la Maison Départementale des Personnes Handicapées (MDPH) est initiée.

- L'observation de poste et l'évaluation de l'ambiance mettent en évidence une grande différence de température $\left(12^{\circ} 2 \mathrm{C}-23^{\circ} 8 \mathrm{C}\right)$ et d’hygrométrie $(29 \%$ - $90 \%)$ entre la plonge et les autres zones de travail, ces variations pouvant être estimées contraignantes; un aménagement de poste avec dispense de plonge est refusé par l'employeur mais des plages de repos avec une hydratation régulière seront respectées.

Elle est revue en visite médicale 6 mois plus tard, son état général s'est amélioré, elle ne se plaint plus de douleurs ni de fatigue et a repris une activité physique quotidienne; elle parvient à respecter les plages de repos avec hydratation régulière conseillées et bénéficie d'un suivi médical adapté dans le cadre de la Reconnaissance de sa Qualité de Travailleur Handicapé (RQTH). Elle ne remplit plus aucun critère selon la nomenclature de Fried. 
Dans cette situation, le repérage a été important car il a permis une orientation adéquate et la mise en confiance de la salariée afin d'expliquer l'intérêt de la RQTH pour son maintien dans l'emploi, la prévention de la désinsertion professionnelle et un suivi médical rapproché. Bien

que l'aménagement optimal n'ait pu être mis en place, la concertation avec le médecin spécialiste a été essentielle pour permettre le respect de consignes déterminantes pour son autonomie et sa qualité de vie, en accord avec l'employeur. [10]

\subsection{Mme R}

Agée de 59 ans, elle occupe un poste de gardienne d'immeuble depuis janvier 2003 et occupe une loge avec son mari âgé de 61 ans, également gardien d'immeuble dans la même résidence.

Elle a pour antécédent notable un cancer du sein gauche opéré avec curage ganglionnaire et traité par radiothérapie microfractionnée il y a quelques mois, pour lequel il existe un lymphoedème et des douleurs séquellaires du sein gauche et bras gauche avec impotence fonctionnelle importante (gauchère), dont le traitement de fond est Anastrazole ${ }^{\circledR}$ et le suivi régulier effectué par un oncologue.

Les symptômes décrits lors de la visite périodique réalisée par l'infirmier en santé au travail, sont une limitation des amplitudes articulaires du bras gauche avec faiblesse de la force de préhension, une perte de poids involontaire, un épuisement, une marche ralentie, une absence d'activité physique, des douleurs diffuses, un œdème majoré du bras gauche

Elle remplit donc 5 critères selon la nomenclature de Fried, ce qui la classe comme « fragile ».

Dans ce contexte, elle est réadressée au médecin du travail afin d'évaluer la situation médicale et de mettre en place un plan d'action : 
- après échange avec l'oncologue référent, un temps partiel thérapeutique est mis en place du fait de la fatigue chronique et des symptômes présentés et une prise en charge globale est décidée avec :

- un avis spécialisé auprès d'un algologue pour améliorer les symptômes de neuropathie séquellaire,

- la prise en charge du lymphoedème par un kinésitherapeute pour drainage lymphatique,

- la prise en charge par une diététicienne afin de permettre une renutrition et une reprise de poids.

- La salariée et son mari sont adressés à l'assistante sociale : il s'avère que son relevé de carrière lui permet de prendre sa retraite pour carrière longue à 60 ans d'ici quelques mois, en même temps que son mari.

Dans le cadre du temps partiel thérapeutique, un aménagement de poste est discuté avec l'employeur : il est convenu d'un aménagement du temps de travail à mi temps et d'une dispense de port de charge et de ménage, ces tâches étant compensées transitoirement par son mari, jusqu'à leur départ à la retraite.

Elle est revue en visite médicale 3 mois après l'aménagement, son état général s'est en partie amélioré, elle a repris du poids et un exercice physique quotidien avec marche 30 minutes/j, la fatigue persiste mais elle est moins marquée, les douleurs ont diminuées, mais il persiste des difficultés de mobilité du membre supérieur avec diminution de la force de préhension ; elle est satisfaite d'avoir pu être maintenue dans son emploi avec des aménagements lui permettant de concilier soins de support et adaptation du poste de travail. Elle remplit à présent deux critères selon la nomenclature de Fried ce qui la classe comme « pré fragile ». 
Dans cette situation, le repérage a été important car il a permis en sensibilisant l'oncologue aux difficultés spécifiques liées au poste de travail une orientation adéquate et l'accompagnement de la salariée afin d'améliorer son confort et sa qualité de vie jusqu'à son départ à la retraite; la coordination de la prise en charge pluridisciplinaire (oncologue, algologue, diététicienne, assistante sociale, médecin du travail) et avec l'employeur a été essentielle. [11]

\subsection{Mr D}

Agé de 52 ans, il occupe un poste d'animateur de secteur/responsable d'entretien de locaux depuis février 1999.

Ses antécédents notables sont un accident de travail en 1996 avec traumatisme de la main gauche et séquelles neuropathiques (pour lequel il a une Reconnaissance de la Qualité de Travailleur Handicapé (RQTH) et une intervention chirurgicale à 2 reprises (2011 et 2016) d'une rupture de la coiffe des rotateurs de l'épaule droite.

Les symptômes décrits lors de la visite périodique réalisée par l'infirmier en santé au travail, sont une absence d'activité physique, une douleur mixte de l'épaule droite avec limitation des amplitudes articulaires et diminution de la force de préhension qui se majore depuis quelques mois, un épuisement du fait d'une douleur insomniante avec répercussions fortes sur le moral, ces symptômes ayant motivé plusieurs arrêts maladie dans les derniers mois, une anxiété liée à la crainte de la perte de son emploi.

Il remplit donc 3 critères selon la nomenclature de Fried, ce qui le classe comme « fragile ».

Dans ce contexte, il est réadressé au médecin du travail afin d'évaluer la situation médicale et de mettre en place un plan d'action : 
- après échange avec l'orthopédiste référent, celui-ci souligne que le port de charges est à limiter et qu'un poste plus sédentaire serait souhaitable ; il envisage du fait de la symptomatologie décrite et des données radiologiques une nouvelle intervention à court terme avec une surveillance post opératoire rapprochée et une rééducation progressive; dans ce cadre, il est décidé avec lui que la reprise se ferait dans le cadre d'un temps partiel thérapeutique, afin de permettre l'accès à la rééducation et le suivi.

- Parallèlement, il est convenu avec le médecin traitant qu'une prise en charge psychologique serait mise en place.

- Une étude de poste par l'ergonome du Service d'Appui au Maintien dans l'Emploi des Travailleurs Handicapés (SAMETH) a permis de discuter les aménagements suivants en lien avec le référent handicap de l'entreprise : déplacements à limiter au maximum (passage d'une fonction multisites à une fonction monosite, durant le temps partiel thérapeutique), véhicule de service avec boite automatique et direction assistée, support d'ordinateur portable avec clavier déporté, souris de type rollermouse, installation d'un logiciel de contrôle sur smartphone, afin de limiter les contraintes au niveau des membres supérieurs et de permettre un meilleur positionnement.

Il est revu sur le lieu de travail par l'ergonome du Service d'Appui au Maintien dans l'Emploi des Travailleurs Handicapés (SAMETH) quelques mois après l'aménagement. Son état général est en partie amélioré, les douleurs sont partiellement soulagées, il persiste une diminution de la force de préhension, le moral est meilleur, la fatigue est toujours présente mais moins marquée, il a repris une activité physique quotidienne et il est satisfait des aménagements mis en place lui permettant de concilier soins et adaptation du poste de travail. Il remplit à présent 2 critères selon la nomenclature de Fried, ce qui le classe comme «préfragile ». 
Dans cette situation, le repérage a été important car il a permis d'améliorer la situation clinique et de sensibiliser le spécialiste afin que le retour à l'emploi soit préparé et se fasse dans les meilleures conditions. Il existait une inadéquation entre l'état de santé du salarié et son poste de travail, entrainant un risque de perte d'emploi ; le service Maintien de Cap Emploi a permis de trouver une solution sur mesure de maintien dans l'entreprise avec aménagement technique du poste. La communication et la collaboration accrue entre les différents acteurs (chirurgien orthopédiste, médecin traitant, ergonome du Service d'Appui au Maintien dans 1'Emploi des Travailleurs Handicapés-(SAMETH), référent handicap de l'entreprise et médecin du travail) a permis une coordination améliorée pour préparer le retour à l'emploi et adapter au mieux son poste de travail afin d'éviter la désinsertion professionnelle. [12]

\section{RESULTATS}

Sur les trois situations cliniques rapportées ci-dessus, nous observons que le repérage de la fragilité, l'orientation pertinente vers le médecin du travail et la coordination d'actions telles que les échanges auprès des spécialistes, l'intervention de l'équipe pluridisciplinaire (assistante sociale, Intervenant en Prévention des Risques Professionnels) et des acteurs du maintien dans l'emploi (référent handicap de l'entreprise, MDPH, SAMETH) ainsi que les échanges avec l'employeur afin de mettre en œuvre les préconisations - même si elles ne sont suivies qu'en partie - pour aménager les postes contribuent à une amélioration de la qualité de vie et du confort ressenti et une prévention de la désinsertion professionnelle.

Notamment, l'action de sensibilisation a occupé une place importante non seulement dans la mise en confiance du salarié pour l'entrée dans la démarche de RQTH et/ou l'adhésion à une meilleure hygiène de vie (activité physique, nutrition...) mais aussi dans l'information des médecins spécialistes pas toujours au fait de l'intérêt de la RQTH et de la mise en place du temps partiel thérapeutique. 
Nous observons également dans ces trois situations une réversibilité du syndrome de fragilité avec dans le premier cas, passage d'un état fragile à un état robuste et dans les deuxième et troisième cas, passage d'un état fragile à un état pré-fragile, ce qui nous laisse penser qu'une action de Santé Publique ciblée sur les adultes d'âge moyen pourrait prévenir, retarder ou inverser les étapes du processus de fragilité.

\section{DISCUSSION}

Sur les trois situations cliniques rapportées ci-dessus, le repérage de la fragilité et la mise en place d'actions correctrices ont permis une amélioration de la qualité de vie et du confort ressenti et une prévention de la désinsertion professionnelle, ce qui illustre bien que le parcours de vie est marqué par la trajectoire robustesse - pré-fragilité - fragilité dépendance, mais que ces derniers états peuvent être prévenus, retardés ou inversés par une action de santé publique ciblée sur les adultes d'âge moyen.

Il a notamment été démontré par l'analyse de 52 études transversales, incluant un suivi de 2,5 à 30 années, la réversibilité de la sarcopénie par l'activité physique et les suppléments nutritionnels. [13]

Ces trois situations éclairent également sur la nécessité que les différents acteurs (salariés, employeurs, médecins de soins) soient parties prenantes de cette approche. Un frein pourrait être par exemple pour le salarié la crainte de perdre son emploi ou le refus du poste aménagé ; pour l'employeur, le manque de possibilités de l'entreprise à aménager un poste ou à trouver un poste compatible; pour les médecins de soins, un refus d'implication. D'où la nécessité pour le médecin du travail d'être disponible pour une coordination et un accompagnement renforcé des différents interlocuteurs. 
La « visite médicale » conseillée à la « retraite » qui marque la fin de l'activité professionnelle est aujourd'hui inadaptée car trop tardive pour adopter une réelle démarche de prévention de la fragilité et des dépendances « évitables ». Comme préconisé en 2015 par l'Académie Nationale de Médecine [14], un bilan de la «fragilité » à mi-vie, dès l'âge de 45-50 ans, puis tous les 5 ans, pour identifier les facteurs de risque de maladies chroniques serait susceptible d'augmenter la durée de vie sans dépendance et d'améliorer la prévention des accidents de travail et des maladies professionnelles. Ceci éviterait une trop forte charge socio-économique de la dépendance au grand âge de la vie et permettrait de garder les personnes au travail en bonne santé plus longtemps.

L'enquête européenne SHARE illustre l'importance de ce problème de santé publique et la nécessité non seulement de détecter le plus précocement possible l'état de fragilité, mais aussi d'intervenir pour éviter l'entrée en dépendance fonctionnelle. Ces interventions sont d'autant plus nécessaires qu'il a été démontré que la sarcopénie et la fragilité sont potentiellement réversibles.

L'étude HEAF démontre que les symptômes de fragilité pourraient probablement être des marqueurs utiles de la difficulté d'emploi à un âge avancé (late middle age) et dans ce cas, par analogie avec les avancées de la médecine gériatrique, ils pourraient servir pour prévenir les pertes de travail liées à la santé et les départs à la retraite anticipés [9]. A l'avenir, Palmer and al. envisagent d'évaluer les relations longitudinales entre les éléments de fragilité en milieu de vie et les données des dossiers médicaux ainsi que les modifications d'état de santé et entre fragilité et changement de statut professionnel. Ils envisagent également d'évaluer les fonctions physiques par des entretiens en face à face, afin d'obtenir des données cliniques plus précises et de suivre leur progression dans le temps.

Finalement, les deux principaux travaux réalisés sur la fragilité des 50 ans et plus ainsi que les 3 cas cliniques présentés mettent en lumière que le concept de fragilité est déjà appréhendé en 
Médecine du Travail et même au-delà, en recouvrant la notion de vulnérabilité telle que décrite par Hervé Michel et Elisabeth Schröder-Butterfill [15]. Pour ces deux auteurs, la fragilité est une notion biomédicale renvoyant à l'analyse des faiblesses intrinsèques d'un individu ; la vulnérabilité est une notion globale, sociétale, relationnelle reposant sur l'analyse des faiblesses d'un individu au regard de son mode de relation avec l'environnement, de ses capacités d'adaptation à un environnement donné et de ses capacités à mobiliser des ressources individuelles et relationnelles.

A partir des résultats observés, nous pouvons imaginer l'ébauche d'un outil de repérage des critères de vulnérabilité des travailleurs en médecine du travail, permettant à l'infirmier(e) en santé au travail le ré-adressage au médecin du travail dès lors que $\underline{3 \text { critères }}$ ont été repérés.

\section{CONCLUSION}

La fragilité représente un réel enjeu de santé publique.

Les résultats prometteurs de l'étude réalisée par Palmer et al. sur la fragilité des travailleurs nous laissent entrevoir que le concept de fragilité développé chez la personne âgée, pourrait être transposé chez la personne plus jeune en activité ; elle pourrait non seulement contribuer à faire adhérer certains médecins de soins à la prise en charge globale et pluridisciplinaire afin de prévenir la désinsertion professionnelle et permettre le maintien dans l'emploi mais aussi certains décideurs en Santé Publique à l'intérêt de la prise en compte de la visite des 45-50 ans.

Les professionnels des services de santé au travail ont un rôle majeur et direct à jouer pour contribuer à l'amélioration de la santé globale de la population en intervenant sur les déterminants de la santé liés directement à l'environnement professionnel (milieu de travail, condition de travail, chômage). 
Aussi, dans le cadre de l'approche globale de la Médecine du_Travail, il est nécessaire de dépasser le concept de fragilité, qui est une notion biomédicale, afin d'y intégrer les facteurs externes que sont notamment la relation à l'environnement de travail, ce qui revient finalement à la définition du concept de vulnérabilité [15].

Les services de santé au travail appliquent déjà en grande partie ce concept : face à une situation de fragilité définie par des déterminants internes qui peuvent être détectés et dépistés de façon préventive, le médecin du travail peut intervenir sur le déterminant externe qu'est l'environnement professionnel et mettre en place des actions afin d'adapter le travail à l'homme.

Ainsi, une détection des situations de vulnérabilité dans le cadre du travail associée à une intervention en amont de la boucle présentéisme/absentéisme/arrêts maladie répétés et/ou prolongés peut favoriser une avancée en âge en meilleure santé et une qualité de vie au travail améliorée.

Le développement d'un outil simple de repérage (tel qu'ébauché à la [figure 3] ) pour identifier les critères de vulnérabilité chez les travailleurs et ainsi concevoir des actions pouvant améliorer leur condition pourrait notamment être mis à disposition des infirmier(e)s en santé au travail afin de faciliter la détection puis l'orientation adaptée vers le médecin du travail.

Travailler plus longtemps et dans les meilleures conditions, prévenir les situations de désinsertion professionnelle en cas de handicap ou au sortir d'une maladie, et prévenir la survenue d'un handicap au grand âge constituent des défis spécifiques qui devraient profiter à tous. 


\section{REFERENCES}

[1] Fried LP. Frailty. In : Hazzard WR, Bierman EL, Blass JP, Ettinger WH, Halter JB, 3rd ed. Principles of geriatric medicine and gerontology. New York: McGraw-Hill, Inc; 1994. p.1149-56.

[2] Lang PO, Michel JP, Zekry D. Frailty syndrome: a transitional state in a dynamic process. Gerontology. 2009;55(5):539-49.

[3] Fried LP, Tangen CM, Walston J, Newman AB, Hirsch C, Gottdiener J, et al. Frailty in Older Adults Evidence for a Phenotype. J Gerontol A Biol Sci Med Sci. 2001 Mar;56(3):M146-57.

[4] Winograd CH, Gerety MB, Chung M, et al. Screening for frailty : criteria and predictors of outcomes. J Am Geriatr Soc. 1991;39:778-84.

[5] Santos-Eggimann B, Cuénoud P, Spagnoli J, Junod J. Prevalence of frailty in middle-aged and older community-dwelling Europeans living in 10 countries. J Gerontol A Biol Sci Med Sci. 2009 Jun;64(6):675-81.

[6] Woo J, Leung J. Multi-morbidity, dependency, and frailty singly or in combination have different impact on health outcomes. Age (Dordr). 2014; 36(2): 923-31.

[7] Macklai NS, et al., Prospective association of the SHARE-operationalized frailty phenotype with adverse health outcomes: evidence from 60+ community-dwelling Europeans living in 11 countries. BMC Geriatr. 2013;13:3.

[8] Borrat-Besson C, Ryser VA, Wernli B. First results from SHARE after the economic crisis Transitions between frailty states - a European comparison, in: Börsch-Supan A, Litwin H, 
Weber G, editor. Active ageing and solidarity between generations in Europe. DE GRUYTER; 2013.p. 175-86.

[9] Palmer KT, D’Angelo S, Harris EC et al. Frailty, prefailty and employment outcomes in Health and Employment After Fifty (HEAF) Study. Occup Med. 2017;74:476-482.

[10] Bachir D., Zemirline F., Niakate A., Cabaret E., Galacteros F. Drépanocytose : le médecin du travail, relais essentiel dans l'information et la prise en charge. Archives des Maladies Professionnelles et de 1'Environnement. 2015;76:373-385.

[11] Mediouni Z. Maladies chroniques et travail. Réunion intersociétés de médecine du travail d’ïle de France 2010 Dec 10. In : INRS, TD 177, DMT N¹26, 2e trimestre 2011; 289-291.

[12] Pichené-Houard A., Fassier J.B., Petit A. Vieillir en bonne santé dans une société du travail. Seconde conférence scientifique sur la prévention et l'intégration du handicap au travail. In : INRS TD 197, Références en Santé au Travail Nº134, juin 2013;106-109.

[13] Cruz-Jentoft AJ, Landi F, Schneider SM, Zuñiga C, Arai H, Boirie Y, Chen LK, Fielding RA, Harris T, Martin FC, Michel JP, Sieber C, Stout JR, Studenski SA, Vellas B, Woo J, Zamboni M, Cederholm T. Prevalence of and interventions for sarcopenia in ageing adults - a systematic review Report of the International Sarcopenia Initiative (EWGSOP and IWGS) Age Ageing; 2014.

[14] Académie Nationale de Médecine. Rapport sur la prévention de la dépendance liée au vieillissement; nov 2015.

[15] Shröder-Butterfill E, Le concept de vulnérabilité et sa relation à la fragilité. In: Michel H, Béland F, editors. La fragilité des personnes âgées, définitions, controverses et perspective d'action. Presses de l'EHESP ; 2013. p.205-228. 
Figure 1 : Le cycle de la fragilité d'après Fried et al. [3]

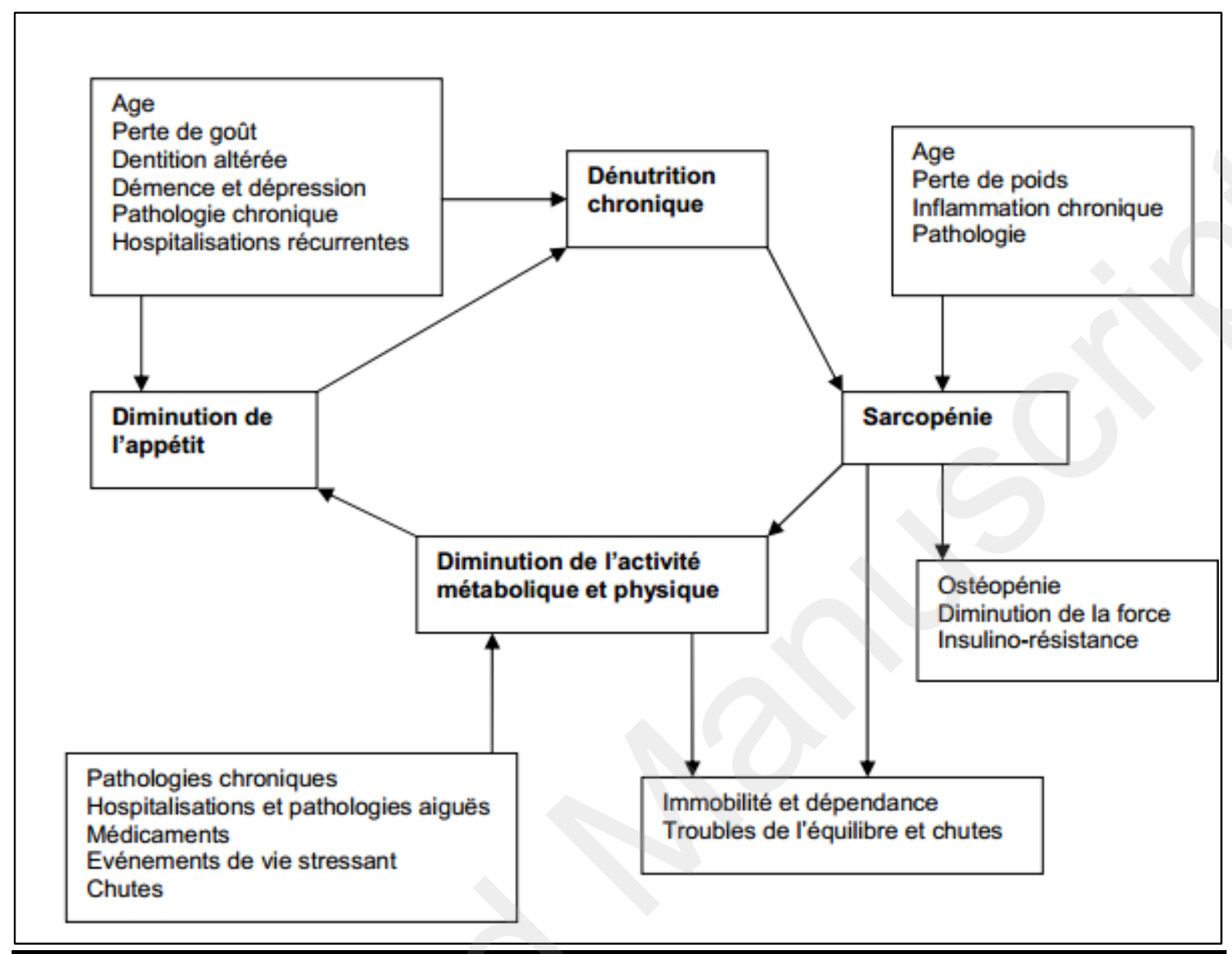

La fragilité est conçue comme un cycle dommageable de processus physiologiques et de déclins physiques dî à des maladies ou des affections qui provoqueront une perte musculaire, suivie de diminution de capacité métabolique qui réduit la mobilité et l'activité physique. La diminution de la dépense énergétique qui s'ensuit amènera à la sous-nutrition chronique qui se résoudra en d'autres pertes de masse musculaire. De ce cycle, cinq composantes du syndrome de fragilité seront proposées : la perte de la force musculaire, le ralentissement de la marche, la diminution de l'énergie, la réduction des activités physiques et la perte involontaire de poids. 
Figure 2 : Schéma du concept de fragilité d'après JP Lang [4]

Le processus de fragilisation se compose de trois états dont la fragilité apparaît comme une étape reconnaissable cliniquement par une récupération lente mais toujours incomplète après la survenue d'une affection aiguë, d'une blessure ou d'un stress. Cet état témoigne de l'insuffisance des réserves homéostatiques qui au stade de pré-fragilité permettraient encore une guérison complète. Le stade ultime du processus est représenté par les complications.

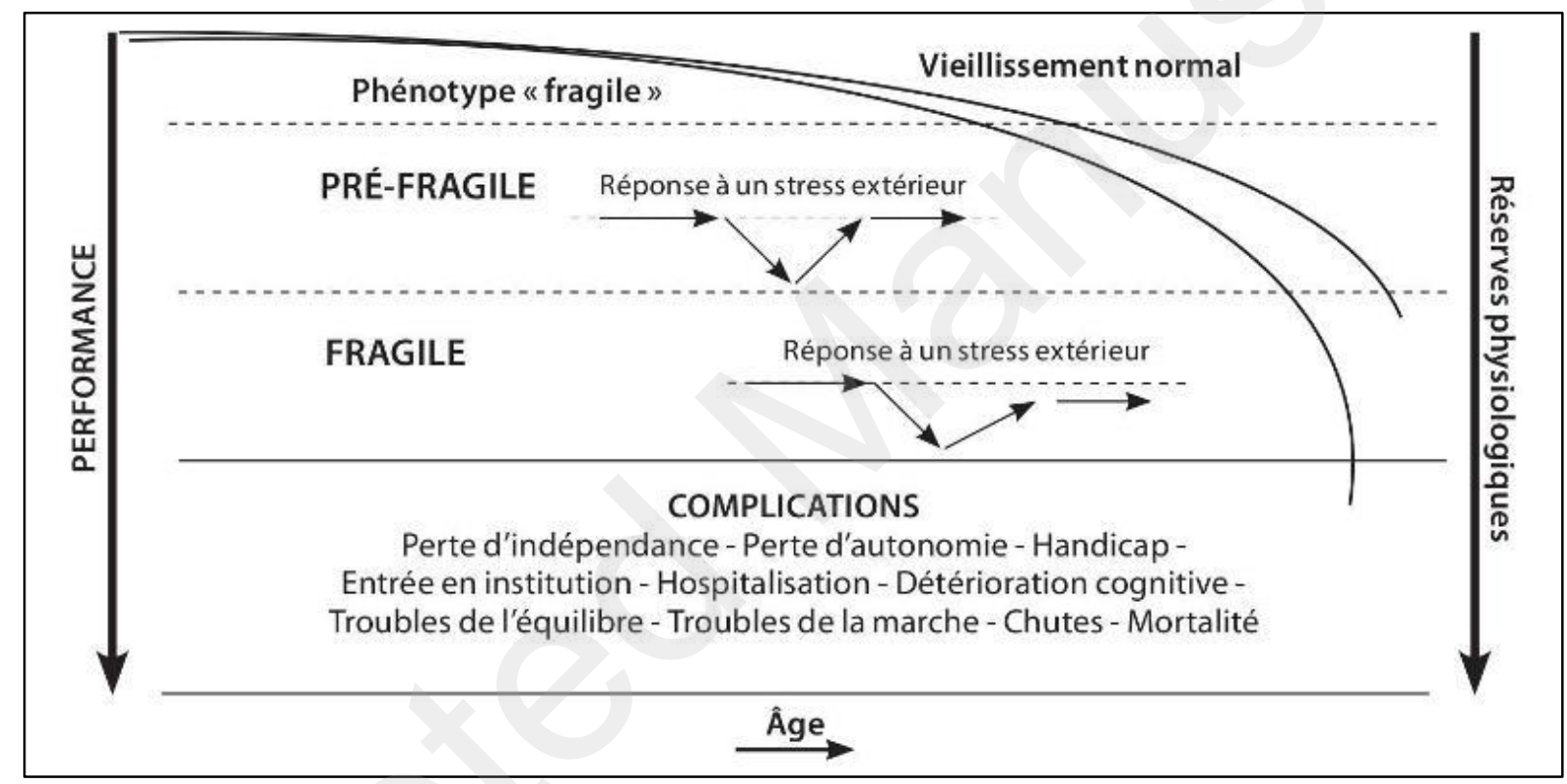


Figure 3 : Ebauche d'un outil de repérage des critères de vulnérabilité des travailleurs en médecine du travail

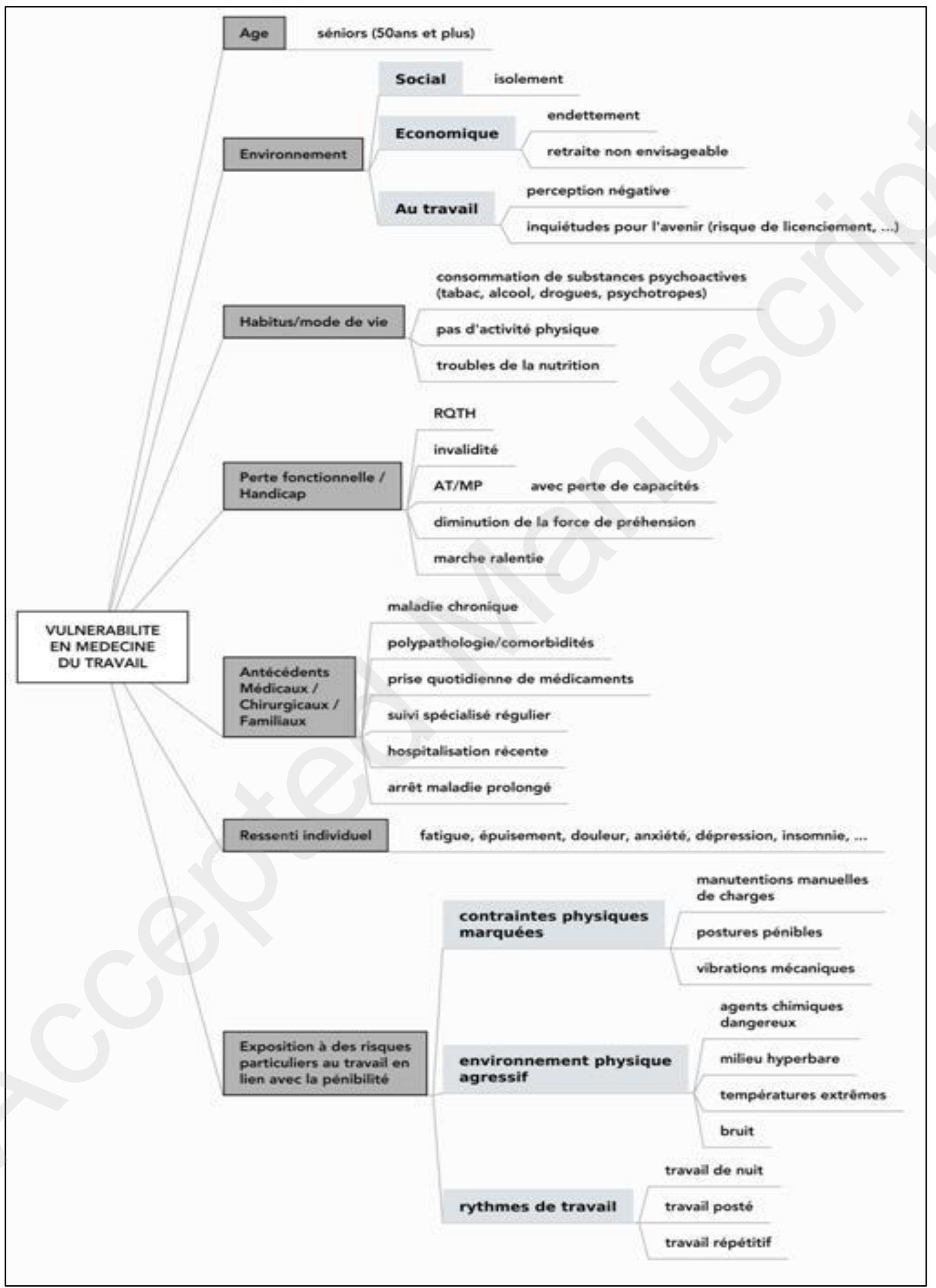

